\title{
Introducción: \\ La psicología educacional: formación, desarrollo y actividad profesional
}

\author{
Introduction: \\ Educational psychology: training, development and professional activity
}

\author{
Alberto Labarrere Sarduy \\ Lourdes Ilizástigui del Portal \\ Universidad Santo Tomás, Santiago, Chile
}

Hoy, como nunca antes, la Psicología educacional es objeto de atención en cuanto a la formación de sus profesionales y la contribución que ellos pueden hacer al desarrollo de la sociedad; en este sentido, el énfasis suele estar puesto también en la capacidad de los psicólogos y psicólogas para enfrentar y resolver tanto problemas teóricos y metodológicos, propios del desarrollo de la disciplina, como aquellos que se refieren a su acción transformadora en los diversos contextos y escenarios sociales.

Es un hecho que la reflexión y el debate concernientes a la Psicología y específicamente la que centra de manera explícita su acción en la educación, han migrado desde el interés en su estatuto científico (Arancibia, Herrera \& Strasser, 2013), hacia la esfera de sus relaciones interdisciplinares y la naturaleza de la actividad que desarrollan los profesionales, así como los problemas que deben resolver (Taborda \& Leoz, 2013; Toro \& Villegas, 2001).

En el sentido antes señalado, resaltan los puntos de vista diversos acerca de las actividades, metodologías e instrumentos de que se vale la Psicología educacional para llevar a cabo su labor de investigación e intervención en los diferentes contextos (Bravo, 2009; Erazo, 2012; Ossa, 2011), se reabre y a la vez alcanza mayor profundidad el debate sobre qué hacen específicamente y qué deben hacer los psicólogos educacionales en dichos contextos; sobre todo hoy, cuando ante el accionar de los profesionales de la Psicología en general, se plantea la exigencia de una actuación más versátil que les facilite enfrentar problemas complejos, con metodologías provenientes de más de una ciencia y en situación de colaboración inter y multidisciplinar (Labarrere, Ilizástigui \& Vargas, 2003).

Respecto a las relaciones interdisciplinares de la Psicología educacional, resulta también foco de controversia la posibilidad de concebir fronteras reales y nítidas con otras disciplinas o áreas, como la Clínica, la Social comunitaria y la Laboral, por sólo mencionar aquellas que en nuestro país disfrutan de un estatuto relativamente más consolidado y alrededor de las cuales se agrupa la mayoría de los psicólogos y psicólogas en predios universitarios y organizaciones gremiales.

Es consenso que al referirse a las diversas disciplinas científicas, se hace sumamente difícil el trazado de límites exactos entre ellas (Morin, 2000), manera de pensar contraria a la representación de que cada una se vale de su arsenal metodológico exclusivo, completamente diferente al de las otras. El problema que se origina entonces, no sólo tiene concomitantes teóricos, epistemológicos y metodológicos, sino también prácticos y de hecho repercute en la formación de los profesionales, los psicólogos entre ellos, así como sobre la manera en que se organizan y ejecutan las tareas propias de su accionar profesional.

Con la publicación de los artículos que componen esta sección especial, se pretende no sólo captar la diversidad de contribuciones que pueden hacer los psicólogos que se identifican como educacionales, sino sobre todo, mostrar algunos tópicos que a nuestro modo de pensar, deberían abordar los psicólogos educacionales de cara al desarrollo no sólo de la Psicología en la Educación, sino además de la Educación chilena.

El primer artículo, ilustra el esfuerzo de un psicólogo educacional por aportar desde lo que podría denominarse como construcción teórico categorial. Eduardo Guzmán, en su trabajo dedicado a la intervención desde una mirada sociocultural, introduce un aspecto sumamente interesante, al proponer que la intervención educacional es "espacio pedagógico de cambio y transformación"; toma decididamente partido en el sentido de que la Psicología educacional se ocupa de la "cultura escolar" y afirma que ello supone la transformación de la Psicología educacional a partir de sus propias construcciones culturales. Aun cuando Guzmán toma como objeto de elaboración categorial 
lo escolar, dando pábulo a la polémica, el llamado que hace a tenerla en consideración presenta, indiscutiblemente, alcances no sólo teóricos sino también epistemológicos y metodológicos. Acaso, es un llamado a revisar prácticas de intervención que, en realidad, constituyen transformaciones "epidérmicas" al no tener en consideración precisa y consecuentemente lo cultural en los contextos de intervención.

El segundo de los trabajos, cuyo autor es José Sebastián Sandoval, corresponde a una investigación que explora el pensar y la actuación de los estudiantes. Ilustra la labor del psicólogo educacional (investigador) que incursionando en el contexto educacional, mediante la indagación científica de fenómenos relevantes para la formación, específicamente de los procesos de enseñanza y aprendizaje. El artículo, dedicado a las concepciones del aprendizaje, estrategias metacognitivas y pericia disciplinar en estudiantes universitarios de Psicología, enlaza tres elementos centrales para comprender el desarrollo del pensamiento y las habilidades de los profesionales en formación, aporta datos sumamente relevantes no sólo para la comprensión del fenómeno investigado, sino además para diseñar estrategias de enseñanza .

Por su parte, el aporte de Ignacio Figueroa Céspedes y María Paz Gómez Montecinos, que de cierto modo podría caracterizarse como una incursión teórico investigativa en la acción de los docentes, aproxima al lector a la importancia de los procesos reflexivos en la práctica de estos profesionales y, muy importante, a su desarrollo profesional. De una manera muy sistemática, los autores evidencian el potencial de la Investigación Acción Transformadora de cara a la promoción de los procesos reflexivos en el docente y de una manera sumamente aguda sitúan al lector ante la evidencia de la necesidad de la reflexión como instrumento de transformación. De manera decidida abogan por el fortalecimiento de los procesos reflexivos y por la necesidad de dejar atrás formas arcaicas de interacción en los contextos educativos (la sala de clases, entre ellos), siendo el pensamiento reflexivo condición e instrumento fundamental para alcanzar este cometido.

Finalmente, Cristóbal Guerra, Cristian Venegas, Luis Ulloa, Hugo Plaza, Andrés Moltedo-Perfetti, con su trabajo dedicado a la validación de un instrumento para medir actitudes en psicólogos y estudiantes de psicología, igualmente se aproximan a una urgencia del desarrollo de nuestra disciplina. Dos aspectos deben destacarse. En primer lugar, la incursión en el campo de las actitudes de estudiantes y profesores, lo que desde el punto de vista práctico constituye un evidente aporte al conocimiento de quienes formamos parte de nuestra disciplina. En segundo orden, pero sólo desde el punto de vista referencial, es muy loable la construcción de un instrumento que, en sí mismo, significa un aporte a la cultura de la Psicología chilena. En un contexto de práctica, donde la mayor parte de los instrumentos con que contamos son foráneos, el diseño y aplicación de instrumentos autóctonos, es crucial; sobre todo si se tienen en cuenta que la mayor parte de los instrumentos (test, pruebas, etc.) de que nos valemos en la práctica cotidiana, son de factura extranjera.

\section{Referencias}

Arancibia, V. H. (2013). Manual de Psicología Educacional (7a ed.). Santiago: Pontificia Universidad Católica de Chile.

Bravo, L. (2009). Psicología educacional, psicopedagogía y educación especial. Revista de investigación en psicología, 12(2), 217-225. Recuperado de http://sisbib.unmsm.edu.pe/bvrevistas/ investigacion_psicologia/v12_n2/pdf/a15v12n2.pdf

Erazo, O. A. (2012). Reflexiones sobre la psicología educativa. Revista de Psicología GEPU, 3(2), 139-157. Recuperado de https://dl.dropboxusercontent.com/u/62290583/Reflexiones\%20 sobre\%201a\%20Psicolog\%C3\%ADa\%20Educativa.pdf

Labarrere, A., Ilizástigui, L. \& Vargas, A. (2003). La formación del psicólogo contribuciones desde una concepción tridimensional. En J. M. Villegas, P. Marassi \& J. P. Toro (Comps.), Problemas centrales para la formación académica y el entrenamiento profesional del psicólogo en las Américas (Vol. 3) (pp. 197-224). Santiago de Chile: Universidad Central.

Morin, E. (2000). El desafio del siglo XXI. Unir los conocimientos. La Paz: Plural.

Ossa, C. (2011). El rol del psicólogo edcacional: la transición desde el paradigma de la simplicidad al paradigma de la complejidad. Revista Pequén, 1(1), 72-82. Recuperado de http://www.ubiobio. cl/miweb/webfile/media/265/el\%20rol\%20del\%20psicologo\%20 educacional.pdf

Taborda, A. \& Leoz, G. (2013). Descripción del objeto y ámbitos de intervención e investigación de la Psicología Educacional. En A. Taborda \& G. Leoz (Comps.), Psicología Educacional en el Contexto de la Clínica Socioeducativa (pp. 31-52). San Luis, Argentina: Nueva Editorial Universitaria.

Toro, J. \& Villegas, J. (2001). Problemas centrales para la formación académica y el entrenamiento profesional del psicólogo en Chile. En J. Toro \& J. Villegas (Eds.), Problemas centrales para la formación académica y el entrenamiento profesional del psicólogo en las Américas (Vol. 1) (pp. 171-228). Buenos Aires: JVE Ediciones. 